factor of ten over that reported by $\mathrm{me}^{3}$ recently in these columns. It is suspected that most of the green line radiation in rapidly interrupted discharges arises in the afterglow, and further experiments are in progress in order to verify this fact.

University of California

JosePH KaPLAN. at Los Angeles.

1 NATURE, 134, 289 ; 1934.

NATURE, 135, 229, Feb. 9, 1935.

\section{Electronic Charge from de Broglie Wave-lengths of Electrons}

Work has been going on for some years at Uppsala for the purpose of increasing the accuracy of the determination of electron wave-lengths. A high-tension set has been arranged, with a special electron valve smoothing circuit taking up changes in input and output, and a tension regulating device including a standard cell, so as to give high tension accurately known and steady within 0.01 per cent. Cathode rays from a hot filament, accelerated by this tension ( $V=15-30 \mathrm{kv}$.) and passing through two narrow slits are diffracted by an etched galena erystal, giving rise to cross-grating diffraction patterns ${ }^{1}$. With this arrangement one gets very sharp spectral lines of different orders. The angles of diffraction can therefore be measured with great accuracy. From these data one can calculate the wave-lengths of the eathode rays with an error less than 0.1 per cent, the grating constant of galena being well known ${ }^{2}$.

Using such wave-length values, one can make interesting calculations of several atomic constants. If, for example, we combine de Broglie's equation,

$$
\lambda \sqrt{1+\frac{e V}{2 m_{0} c^{2}}}=\frac{h}{\sqrt{ } 2 e m_{0} V}=\lambda^{\prime},
$$

with the expression for the Rydberg constant,

$$
R=\frac{2 \pi^{2} e^{4} m_{0}}{c h^{3}},
$$

we obtain for the calculation of the electronic charge

$$
e=\text { const. } \frac{V^{3 / 4} \lambda^{/ 3 / 2}}{\sqrt[4]{e} / m_{0}}
$$

$R$ is known from spectroscopic measurements with very great accuracy, and $e / m_{0}$ is fairly well known and enters the formula in the power $1 / 4$. Thus, we can hope to find $e$ with an accuracy sufficient to enable us to choose between oil drop and X-ray values of $e$.

Preliminary measurements give $e=(4 \cdot 796 \pm 0 \cdot 010)$ $\times 10^{-10}$ E.S.U.

The work is proceeding and a definite and detailed report will shortly be published elsewhere.

Physics Laboratory,

$$
\text { S. v. Friesen. }
$$

University, Uppsala. May 22.

${ }^{1}$ S. v. Friesen, Arkiv f. Mat. Astr. o. Fys., 24B, No. 8; 1934.

${ }^{2}$ E. v. Zeipel, ibid., 25A, No. 8; 1935.

\section{A Search for the Extreme Infra-Red Spectrum of the Sun}

THE solar spectrum in the infra-red has been thoroughly investigated throughout the range of wavelengths up to about $11 \mu$. Radiation of longer wavelength is practically completely absorbed, chiefly by the water vapour present in the atmosphere. Accord. ing to $\mathrm{E}$. von $\mathrm{Bahr}^{1}$, there are reasons to expect, from theoretical considerations, that for wavelengths greater than $c a .400 \mu$, water vapour will be again transparent. In 1914 Rubens and Schwarzschild $^{2}$ made an attempt at the Astrophysical Observatory at Potsdam to measure the intensity of solar radiation in this region of the spectrum by using the quartz lens method for isolating it, but the intensity proved too small to be detected even by means of the very sensitive instrument they used.

On the suggestion of Prof. M. A. Lewitsky, we made a similar attempt last summer in the exceptionally clear atmosphere of the southern slopes of Mount Elbruz (Caucasus) at an altitude of $3,000 \mathrm{~m}$. above sea-level. For isolating the extreme infra-red radiation, we used two sheets of black paper placed in front of a metal mirror $60 \mathrm{~cm}$. in diameter; this focused the radiation transmitted through the paper on to one group of junctions of a differential surface thermopile, which was used in order to minimise the otherwise very considerable influence of stray radiation and changes of temperature. The thermocurrent was measured by means of a photo-relay similar in design to that described by Bergmann ${ }^{3}$. For illuminating the differential photo-cell of this relay we used a heliostat.

The apparatus proved so sensitive as to enable us to record one tenth of the radiation that was to be expected according to Planck's law for the region beyond $400 \mu$; that is, the only region of wavelengths likely to pass through the water vapour of atmosphere, two sheets of black paper and a quartz window, $2 \mathrm{~mm}$. in thickness, of the thermopile.

Since the layer of the atmosphere below $3,000 \mathrm{~m}$ holds about five-sixths of the total amount of water vapour contained in the atmosphere, we may take it that, so far as the absorption of the sun's rays by water vapour is concerned, the conditions of our experiment were about five times as favourable as those of Rubens and Schwarzschild, who worked at low altitude and in a contaminated atmosphere. On the other hand, the sensitivity of their apparatus was about twice as great as that possible with our apparatus in the open air. Although we had thus, on the whole, somewhat better conditions than they, we also were unable to detect any trace of radiation from the sun in the extreme infra-red.

A detailed description of our experiment will be published elsewhere.

$$
\text { V. G. VAFIAdi. }
$$

Optical Institute,

S. S. KRIVICH. Leningrad.

G. V. Pokrovsky.

1 E. von Bahr, Verh. Deutsch. Phys. Ges., 710; 1913. Rubens und Schwarzschild, Sitzungsberichte Preus. Akad. Wissensch., $702 ; 1914$.

${ }^{3}$ L.Bergmann, Phys. Z., 32, 688 ; 1931.

\section{Philosophy and Modern Science}

IT is disconcerting to find how rarely physical scientists trouble to make themselves familiar with the results of recent psychological investigation, even those results of the more important and far-reaching 